\title{
INTERVENCIÓN EN FUNCIONES EJECUTIVAS EN EDUCACIÓN INFANTIL
}

\author{
Miriam Romero López \\ miriam@ugr.es \\ Alicia Benavides Nieto \\ María Fernández Cabezas \\ M. Carmen Pichardo Martínez \\ Universidad de Granada \\ https://doi.org/10.17060/ijodaep.2017.n1.v3.994
}

Fecha de Recepción: 20 Enero 2017

Fecha de Admisión: 1 Abril 2017

\section{RESUMEN}

Las funciones ejecutivas definidas como el conjunto de procesos cognitivos de orden superior que permiten un comportamiento intencional y dirigido hacia un objetivo, son vitales para el éxito en la vida, en la escuela, y en el trabajo. Mejorar las funciones ejecutivas en las primeras etapas es muy importante porque los problemas en el funcionamiento ejecutivo, en la etapa preescolar, predicen los logros cognitivos en etapas posteriores y tienen un papel central en la preparación escolar y en la adquisición de las primeras habilidades académicas. Estudios recientes han encontrado relaciones positivas entre las capacidades de funcionamiento ejecutivo y la preparación de matemáticas en niños en edad preescolar. Igualmente, una extensa bibliografía sugiere una relación inversa entre las funciones ejecutivas y la conducta agresiva en los seres humanos. Por esta razón, el objetivo de la presente investigación es realizar una revisión de los programas y actividades destinados a la mejora de las funciones ejecutivas en la etapa preescolar. Diversas actividades y programas han demostrado mejorar las funciones ejecutivas de los niños, tales como los juegos computarizados, las artes marciales, el yoga, la atención plena y los programas incluidos en el currículum escolar. Los resultados de los estudios realizados sobre la aplicación de algunas de las actividades o programas mencionados son prometedores y sugieren que las diferentes estrategias pueden ser útiles para mejorar las funciones ejecutivas durante el período preescolar.

Palabras clave: funciones ejecutivas, educación infantil, intervención, desarrollo, control inhibitorio, memoria de trabajo, flexibilidad cognitiva.

\section{ABSTRACT \\ Intervention in executive functions in early childhood education \\ Executive functions defined as the set of higher-order cognitive processes that allow an inten- tional and directed behavior towards a goal, are vital for success in life, at school, and at work.}




\section{INTERVENCIÓN EN FUNCIONES EJECUTIVAS EN EDUCACIÓN INFANTIL}

Improving executive functions in the early stages is very important because problems in executive functioning, in the preschool stage, predict cognitive achievement in later stages and have a central role in school readiness and in the first academic skills acquisition. Recent studies have found positive relationships between executive functioning capacities and math readiness in preschoolers. Likewise, an extensive bibliography suggests an inverse relationship between executive functions and aggressive behavior in humans. For this reason, the objective of this research is to conduct a review of the programs and activities aimed at improving the executive functions in the preschool stage. Various activities and programs have been shown to improve children executive functions, such as computer games, martial arts, yoga, mindfulness and programs included in the school curriculum. The results of studies conducted on the implementation of some of the above activities or programs are promising and suggest that different strategies can be useful in improving executive functions during the preschool years.

Key words: executive functions, early childhood education, evaluation, Intervention, development, inhibitory control, working memory, cognitive flexibility.

\section{ANTECEDENTES}

La edad preescolar es una etapa vital para el desarrollo cognitivo, social, emocional y físico de los niños. Durante este periodo, que en las sociedades occidentales generalmente abarcan de los 3 a los 6 años de edad, los niños desarrollan algunas de las principales cualidades que serán claves para el éxito en la vida y en la escuela (van Lier y Deater-Deckard, 2016). Esas cualidades son conocidas como funciones ejecutivas, definidas como el conjunto de procesos cognitivos de orden superior que permiten controlar los comportamientos esenciales para la realización de tareas y el aprendizaje, y que contribuyen a la regulación y supervisión de las mismas (Baggetta y Alexander, 2016).

En los niños, las funciones ejecutivas no sólo son necesarias para regular el comportamiento en situaciones sociales 0 académicas, sino también para controlar los comportamientos impulsivos y seguir las instrucciones de los profesores o de sus progenitores. Muchos niños tienen dificultades para frenar su conducta y no tocar los juguetes atractivos en una tienda a pesar de la prohibición de sus padres, no pueden abstenerse de hacer comentarios inapropiados en lugares públicos, o tienen dificultad para esperar su turno al jugar a un juego. En todas estas situaciones, los niños presentan déficits en su control inhibitorio.

Por otro lado, cuando los maestros piden en una clase que los niños realicen un ejercicio, los niños primero tendrán que prestar atención a lo que el profesor dice y presentar una buena memoria de trabajo para recordar las distintas instrucciones. Cuando se presenta otro ejercicio diferente, los niños necesitan una buena flexibilidad cognitiva para pasar de una tarea a otra totalmente diferente. Igualmente, la flexibilidad favorece que los niños tengan en cuenta diferentes perspectivas y piensen distintas soluciones que no habían considerado.

Las funciones ejecutivas son, pues, necesarias en muchas situaciones. Se desarrollan durante la infancia y maduran en la adolescencia, pero el crecimiento más significativo se produce en el período preescolar. Por tanto, es particularmente interesante observar este período de desarrollo (Volckaert y Noël, 2015).

En los últimos años, se han propuesto varios programas de formación destinados a la mejora de las funciones ejecutivas (Diamond y Lee, 2011). Aunque aún existen muchas preguntas sin respuestas relacionadas con la eficacia de las intervenciones en funciones ejecutivas (Melby-Lervag y Hulme, 2013; Shipstead, Redick, y Engle, 2012), lo que si parece claro es que las funciones ejecutivas se pueden mejorar desde etapas tempranas.

Sin embargo, aunque los programas destinados a la mejora de las funciones ejecutivas en la primera infancia muestran resultados muy positivos, la mayoría de los estudios se centran en niños en 
edad escolar o universitarios (por ejemplo, Chen, Yue, Tian, y Jiang, 2016; Dias y Seabra, 2016). A pesar del potencial efecto preventivo de la intervención temprana, hasta el momento, sólo un número limitado de estudios han investigado el efecto de la intervención de las funciones ejecutivas en niños en edad preescolar (3-5 años) (Di Lieto et al., 2017; Sonuga-Barke y Halperin, 2011).

En esta línea, muchos niños comenzarán la escuela sin la madurez y los comportamientos emocionales y sociales necesarios para aprender, generando problemas en el rendimiento escolar a corto plazo y en las tasas de graduación en secundaria a largo plazo (Bierman, Nix, Greenberg, Blair, y Domitrovich, 2008). No obstante, tal y como defiende Diamond (2013) y Melby-Lervag y Hulme (2013) los niños más pequeños pueden obtener beneficios significativamente más elevados de los programas de formación que los niños en edad escolar y estos beneficios pueden favorecer la preparación para la etapa escolar (Blair, 2002).

En cuanto a las intervenciones en edad preescolar, es difícil comparar los efectos de los distintos programas de intervención debido a que difieren en su duración (intervenciones a largo versus corto plazo), el tipo de agrupamiento (individuales versus intervenciones grupales) y los materiales (Traverso, Viterbori, y Usai, 2015).

Los programas a largo plazo son en general, intervenciones grupales realizadas en la escuela, que se ofrecen a lo largo de los tres años de Educación Infantil o durante el año anterior a la entrada de la escuela primaria (por ejemplo, Raver, Jones, Li-Grining, Zhai, Bubb, y Pressler, 2011).

La implementación de los programas a largo plazo requieren una serie de requisitos muy estrictos y presentan una serie de inconvenientes. En primer lugar, es necesario mucho tiempo para llevarlos a cabo. Además, se necesita contar con el compromiso de los directores de las escuelas, así como con el interés, la formación docente y una buena relación docente-alumno (Domitrovich, Cortes, y Greenberg, 2007). La necesidad de estos recursos hace que estos programas a veces resulten caros y poco viables.

Por otro lado, las intervenciones a corto plazo son generalmente formaciones individualizadas cuya duración suele ir desde una semana a un mes. Este tipo de entrenamiento suele basarse en tareas computarizadas con horarios intensivos de dos a cinco sesiones por semana (BergmanNutley, Soderqvist, Bryde, Thorell, Humphreys, y Klingberg, 2011; Thorell, Lindqvist, Bergman Nutley, Bohlin, y Klingberg, 2009) o actividades de papel y lápiz con tres a ocho sesiones cortas concentradas en una semana (Kloo y Perner, 2003). Sin embargo, estos programas rara vez son generalizables a las actividades de la vida diaria, presentando escasa validez ecológica. Además, muchos de ellos también requieren de una extensa formación del profesorado (Bergman-Nutley et al., 2011; Thorell et al., 2009).

Aunque la mayoría de las intervenciones pueden tener efectos positivos sobre la población en la que actúan, Diamond (2014) establece 5 principios necesarios para el éxito de los programas de intervención en funciones ejecutivas: (1) cuanto más exigentes sean las tareas de funciones ejecutivas mayor será la diferencia entre el grupo experimental y el control. Por tanto, es necesario poner al límite las habilidades ejecutivas de los niños para favorecer su desarrollo (Davis, Marra, Najafzadeh, y Lui-Ambrose, 2011; Diamond, Barnett, Thomas, y Munro, 2007); (2) a veces, la formación en las funciones ejecutivas puede transferirse a otros campos no trabajados, pero algunos estudios demuestran que la transferencia de un ámbito a otro, generalmente, es muy estrecha (Bergman-Nutley et al., 2011); (3) los niños que presentan mayores déficits en funciones ejecutivas (incluidos los niños desfavorecidos) se benefician más de cualquier intervención o programa destinado a la mejora de las funciones ejecutivas (Flook et al., 2010; Karbach y Kray, 2009); (4) los programas de entrenamiento en funciones ejecutivas deben ir incrementando en dificultad o no se obtendrán beneficios (Bergman-Nutley et al., 2011; Holmes, Gathercole, y Dunning, 2009). Si la difi- 
cultad no aumenta, la actividad se vuelve monótona y aburrida y los niños pierden el interés (Diamond, 2014); (5) la práctica repetida es clave. La mejora en funciones ejecutivas depende de la cantidad de tiempo que se dedique a trabajar estas habilidades.

Por tanto, la presente revisión tiene como objetivo analizar los diferentes programas destinados a la mejora de las funciones ejecutivas en la infancia y conocer la importancia de elaborar programas para su mejora desde etapas tempranas.

\section{PROGRAMAS DE INTERVENCIÓN TEMPRANA BASADOS EN LA PROMOCIÓN DE LAS FUNCIONES EJECUTIVAS}

\section{Programas escolares}

Uno de los programas con mayor repercusión es el programa Tools of the Mind: The Vygotskian Approach to Early Childhood Education (Bodrova y Leong, 2007). Este programa tiene como fin la mejora de las funciones ejecutivas en la infancia a través de actividades que consisten en el juego dramático, la regulación del habla y juegos de memoria y atención. Estudios que han probado la eficacia de este programa, han demostrado que los niños y niñas que participan en él, no solo mejoran las funciones ejecutivas sino que, estas mejoras se generalizan a otros ámbitos como el comportamiento social 0 el rendimiento académico. En un ensayo aleatorio, Diamond et al. (2007) encontraron que los niños en edad preescolar con familias con bajos ingresos que participaron en el programa programa Tools of the Mind: The Vygotskian Approach to Early Childhood Education mostraron un mejor rendimiento en funciones ejecutivas que el grupo control.

Otro programa muy relevante en la mejora de las funciones ejecutivas en la infancia es el programa Montessori (Lillard y Else-Quest, 2006). Ambos programas comparten una serie de características comunes: (a) ayudan a que los niños ejerzan control sobre sus funciones ejecutivas y las actividades van incrementando su en dificultad; (b) reducen el estrés en el aula; (c) rara vez avergüenzan a un niño; (d) cultivan la alegría, el orgullo y la autoestima de los niños; (e) adoptan una actitud activa y enfoque práctico para el aprendizaje; (f) se acomodan a los diferentes ritmos a los que progresan los niños; $(\mathrm{g})$ hacen hincapié en el desarrollo del carácter, así como el desarrollo académico; (h) hacen énfasis en el lenguaje oral; (i) involucran a los niños en la enseñanza entre compañeros; (j) y trabajan las habilidades sociales.

Muchas de estas características son también compartidas con otros dos programas escolares destinados a la etapa preescolar: Promoting Alternative Thinking Strategies (PATHS, Riggs, Greenberg, Kusche, y Pentz, 2006) y el programa Chicago School Readiness Project (CSRP, Raver et al., 2011). El programa PATHS capacita a los maestros para que fomenten el desarrollo en los niños del autocontrol, el reconocimiento y manejo de los sentimientos, y la resolución de problemas interpersonales. Los niños pequeños experimentan y reaccionan a las emociones antes de que puedan verbalizar lo que les pasa, y a menudo reaccionan impulsivamente y sin control. Por lo tanto, la formación en la verbalización de los sentimientos y la práctica de estrategias de autocontrol consciente (por ejemplo, esperar antes de actuar y el diálogo interno) son algunas de las habilidades que se obtienen. Cuando los niños se molestan, deben parar, realizar una respiración profunda, decir cuál es el problema y cómo se sienten, y posteriormente, construir un plan de acción. Los maestros utilizan técnicas para enseñar a los niños a generalizar las habilidades aprendidas durante el programa PATHS a otros contextos. Después de un año participando en el programa, los niños en edad preescolar mostraron un mejor control inhibitorio y flexibilidad cognitiva que los niños del grupo control. A su vez, los niños que mostraron un mayor control inhibitorio en la fase post-test mostraron menos internalización y externalización de los problemas de comportamiento (Riggs et al., 2006). 
Por su parte, el programa Chicago School Readiness Project (CSRP) tiene como objetivo mejorar las posibilidades de éxito en la escuela de los niños en edad preescolar con familias con bajos ingresos. Para ello, se trabaja el ajuste emocional y conductual de los niños pequeños a través de una intervención integral, implementada en el aula. Los niños en edad preescolar con un nivel socioeconómico bajo que participaron en el programa CSRP mostraron mejores resultados en funciones ejecutivas que sus iguales del grupo control. Igualmente, en los tres años siguientes, obtuvieron mejores resultados que los controles en matemáticas y en lectura y esos logros académicos fueron mediados por mejoras en las funciones ejecutivas (Li-Grining, Raver, y Pess, 2011).

\section{Entrenamiento computarizado}

Uno de los programas computarizados más utilizados es el Cogmed Working Memory Training, basado en la mejora de la memoria de trabajo. El programa de entrenamiento contiene 25 sesiones de entrenamiento, de 30-40 minutos por sesión. Tiene una duración total de 5 semanas, con 5 sesiones por semana. El programa completo incluye una entrevista inicial, una sesión de inicio, 5 semanas de entrenamiento con llamadas semanales del entrenador, acceso a la red de entrenamiento Cogmed, sesión de conclusión final del programa, entrevista semestral de seguimiento y entrenamiento Cogmed ampliado. Diversos estudios han demostrado que los niños que participan en el programa Cogmed Working Memory Training obtuvieron puntuaciones más elevadas en memoria de trabajo que sus iguales del grupo control (por ejemplo, Holmes y Gathercole, 2014; Thorell et al., 2009). Los resultados sugieren que el entrenamiento de la memoria de trabajo puede tener efectos significativos desde la etapa preescolar.

Sin embargo, a pesar de que existen evidencias de que el entrenamiento computarizado puede mejorar la memoria de trabajo de los niños, hasta el momento los intentos de mejorar el control inhibitorio en niños de 4 a 6 años de edad utilizando juegos computarizados, no han tenido éxito (Rueda, Rothbart, McCandliss, Saccomanno, y Posner, 2005; Thorell et al., 2009). No obstante, otras formas de entrenamiento, como por ejemplo los programas escolares anteriormente comentados mejoran la inhibición en niños de 4 a 6 años de edad. Por tanto, puede ser que la formación computarizada no sea la mejor opción para el entrenamiento del control inhibitorio en niños tan pequeños, o bien puede que aún no se haya encontrado la manera de utilizar de forma óptima las computadoras para mejorar el control inhibitorio (Diamond y Lee, 2011).

\section{Actividad física y funciones ejecutivas}

Los bajos niveles de actividad física son comunes en adultos y niños, tanto es así, que se ha producido recientemente un llamamiento a la acción de la "pandemia de la inactividad física" (Kohl et al., 2012). La actividad física es bien conocida por ser beneficiosa para la salud física. Sin embargo, la evidencia también sugiere que está relacionada con la salud mental, incluyendo la función cognitiva, en poblaciones con y sin dificultades psicológicas (Booth et al., 2013).

En las últimas décadas, numerosos estudios han examinado la relación entre la actividad física y el funcionamiento cognitivo. Los resultados recientes indican que la actividad física no sólo mejora el funcionamiento cognitivo en general, sino que también mejora el rendimiento en tareas que requieren de las funciones ejecutivas (Donnelly et al., 2016; Khan y Hillman, 2014; Tomporowski et al., 2015).

Un estilo de vida físicamente activo durante la infancia parece tener una influencia positiva en el desarrollo del cerebro. En esta línea, Aadland, Moe, Aadland, Anderssen, Resaland, y Ommundsen (2017) realizaron un estudio en el que participaron 697 niños y encontraron fuertes asociaciones entre las habilidades motoras y las funciones ejecutivas. 


\section{INTERVENCIÓN EN FUNCIONES EJECUTIVAS EN EDUCACIÓN INFANTIL}

En un estudio piloto realizado con niños de 10 a 13 años sobre los efectos del yoga (que implicaban el entrenamiento físico, la relajación y la conciencia sensorial) en las funciones ejecutivas de los niños, se encontró que aquellos niños que practicaron el yoga 75 minutos al día, 7 días a la semana durante 1 mes, mejoraron su planificación y su flexibilidad cognitiva en comparación con aquellos niños que no habían realizado yoga (Manjunath y Telles, 2001).

Igualmente, Lakes y Hoyt (2004) realizaron un estudio en el que asignaron al azar a niños de 5 a 11 años a dos grupos: uno que realizaba educación física tradicional y otro que realizaba taekwondo. Los niños que realizaron taekwondo grupo obtuvieron mejores resultados en memoria de trabajo y control inhibitorio que aquellos que realizaron educación física tradicional.

Por tanto, las diferentes dimensiones del desempeño cognitivo, tales como la velocidad de procesamiento, la planificación y las estrategias de control, y la memoria de trabajo, se podrían mejorar con el ejercicio físico y la actividad física regular.

\section{METODOLOGÍA}

Estudio de revisión bibliográfica de la literatura existente sobre la temática.

\section{RESULTADOS}

Partiendo de la revisión teórica realizada parece importante la elaboración de programas preventivos y de intervención que favorezcan el desarrollo de las funciones ejecutivas en niños pequeños, puesto que el funcionamiento ejecutivo en la infancia predice el rendimiento durante toda la vida y se ha relacionado con la mejora de la competencia social, la prevención de los problemas de conducta, el éxito académico, la mejora de la salud y la calidad de vida.

\section{DISCUSIÓN Y CONCLUSIONES}

Los resultados de los estudios anteriores sobre intervenciones en funciones ejecutivas son muy positivos y sugieren que las funciones ejecutivas se pueden mejorar desde la etapa preescolar mediante la utilización de diferentes estrategias. Sin embargo, la mayoría de los programas de intervención en funciones ejecutivas están destinados a niños en la etapa escolar y muchos de los estudios tratan sobre la efectividad de las intervenciones en un conjunto limitado de las funciones ejecutivas, por ejemplo, se centran únicamente en componentes específicos de las funciones ejecutivas, como la memoria de trabajo.

No obstante, a pesar de estas limitaciones, la intervención temprana en funciones ejecutivas es necesaria puesto que diversos estudios han demostrado que los beneficios de las intervenciones tempranas de alta calidad se pueden mantener en los años escolares posteriores e incluso en la edad adulta, particularmente en niños con familias de bajos ingresos (Blair y Raver, 2014; Donnelly et al., 2016; Tomporowski et al., 2015).

\section{AGRADECIMIENTOS}

Investigación realizada gracias a la ayuda para la formación de profesorado universitario (FPU13/03470) del Ministerio de educación, cultura y deporte.

\section{REFERENCIAS}

Aadland, K. N., Moe, V. F., Aadland, E., Anderssen, S. A., Resaland, G. K., y Ommundsen, Y. (2017). Relationships between physical activity, sedentary time, aerobic fitness, motor skills and executive function and academic performance in children. Mental Health and Physical Activity, 12, 1018.

Baggetta, P., y Alexander, P. A. (2016). Conceptualization and Operationalization of Executive Function. Mind, Brain, and Education, 10(1), 10-33. 
Bergman-Nutley, S., Soderqvist, S., Bryde, S., Thorell, L. B., Humphreys, K., y Klingberg, T. (2011). Gains in fluid intelligence after training non-verbal reasoning in 4-year-old children: a controlled, randomized study. Developmental Science, 14, 591-601. doi: 10.1111/j.14677687.2010.01022.x

Bierman, K. L., Nix, R. L., Greenberg, M. T., Domitrovich, C. E., y Blair, C. (2008). Executive functions and school readiness intervention: impact, moderation, and mediation in the head startREDI program. Development and Psychopathology. 20, 821-843. doi: $10.1017 /$ S0954579408000394.

Blair, C. (2002). School readiness: integrating cognition and emotion in a neurobiological conceptualization of child functioning at school entry. American Psychologist, 57, 111-127. doi: 10.1037/0003-066X.57.2.111.

Blair, C., y Raver, C. C. (2014). Closing the achievement gap through modification of neurocognitive and neuroendocrine function: Results from a cluster randomized controlled trial of an innovative approach to the education of children in kindergarten. PLoS One, 9, 112-393. doi:10.1371/journal.pone. 0112393

Bodrova, E., y Leong, D. J. (2007). Tools of the Mind: The Vygotskian Approach to Early Childhood Education. New York: Pearson.

Booth, J. N., Tomporowski, P. D., Boyle, J. M., Ness, A. R., Joinson, C., Leary, S. D., y Reilly, J. J. (2013). Associations between executive attention and objectively measured physical activity in adolescence: findings from ALSPAC, a UK cohort. Mental Health and Physical Activity, 6(3), 212219.

Chen, T. T., Yue, G. H., Tian, Y. X., y Jiang, C. H. (2016). Baduanjin Mind-Body Intervention Improves the Executive Control Function. Frontiers in Psychology, 7, 1-8. doi: 10.3389/fpsyg.2016.02015

Davis, J. C., Marra, C. A., Najafzadeh, M., y Lui-Ambrose, T. (2011). The independent contribution of executive functions to health related quality of life in older women. BMC Geriatrics, 10, 16-23.

Diamond, A. (2012). Activities and programs that improve children's executive functions. Current directions in psychological science, 21(5), 335-341.

Diamond, A. (2013). Executive Functions. Annual Review of Psychology, 64, 135-168. doi: 10.1146/annurev-psych-113011-143750.

Diamond, A. (2014). Want to Optimize Executive Functions and Academic Outcomes?: Simple, Just Nourish the Human Spirit. En Minnesota Symposia on Child Psychology (Vol. 37, p. 205). NIH Public Access.

Diamond, A., Barnett, W. S., Thomas, J., y Munro, S. (2007). Preschool program improves cognitive control. Science, 318, 1387-1388.

Diamond, A., y Lee, K. (2011). Interventions shown to aid executive function development in children 4 to 12 years old. Science, 333, 959-964.

Dias, N. M., y Seabra, A. G. (2016). Intervention for executive functions development in early elementary school children: effects on learning and behaviour, and follow-up maintenance. Educational Psychology, 1-19.

Di Lieto, M. C., Inguaggiato, E., Castro, E., Cecchi, F., Cioni, G., Dell'Omo, M., ... y Dario, P. (2017). Educational Robotics intervention on Executive Functions in preschool children: A pilot study. Computers in Human Behavior, 71, 16-23.

Donnelly, J. E., Hillman, C. H., Castelli, D., Etnier, J. L., Lee, S., Tomporowski, P., ... y Szabo-Reed, A. N. (2016). Physical activity, fitness, cognitive function, and academic achievement in children: A systematic review. Medicine \& Science in Sports \& Exercise, 48(6), 1197e1222. http://dx.doi.org/10.1249/ mss.0000000000000901. 
Domitrovich, C. E., Cortes, R., y Greenberg, M. T. (2007). Improving young children's social and emotional competence: a randomized trial of the preschool PATHS program. The Journal of Primary Prevention, 28, 67-91. doi: 10.1007/s10935-007-0081-0.

Flook, L., Smalley, S. L., Kitil, M. J., Galla, B. M., Kaiser-Greenland, S., Locke, J., ... y Kasari, C. (2010). Effects of mindful awareness practices on executive functions in elementary school children. Journal of Applied School Psychology, 26(1), 70-95.

Holmes, J., y Gathercole, S. E. (2014). Taking working memory training from the laboratory into schools. Educational Psychology, 34(4), 440-450.

Holmes, J., Gathercole, S. E., y Dunning, D. L. (2009). Adaptive training leads to sustained enhancement of poor working memory in children. Developmental science, 12(4), 9-15.

Karbach, J., y Kray, J. (2009). How useful is executive control training? Age differences in near and far transfer of task switching training. Developmental science, 12(6), 978-990.

Khan, N. A., y Hillman, C. H. (2014). The relation of childhood physical activity and aerobic fitness to brain function and cognition: A review. Pediatric Exercise Science, 26(2), 138e146. http://dx.doi.org/10.1123/pes.2013-0125.

Kloo, D., y Perner, J. (2003). Training transfer between card sorting and false belief understanding: helping children apply conflicting descriptions. Child Development, 74, 1823-1839. doi: 10.1046/j.1467-8624.2003.00640.x.

Kohl, H. W., Craig, C. L., Lambert, E. V., Inoue, S., Alkandari, J. R., Leetongin, G., ... y Kahlmeier, S. (2012). The pandemic of physical inactivity: global action for public health. The Lancet, 380(9838), 294-305.

Lakes, K. D., y Hoyt, W. T. (2004). Promoting self-regulation through school-based martial arts training. Applied Developmental Psychology, 25, 283-302.

Li-Grining, C. P., Raver, C. C., y Pess, R. A. (2011). Academic impacts of the Chicago School Readiness Project: Testing for evidence in elementary school. Paper presented at the Biennial Meeting of the Society for Research in Child Development, Montreal, QC, Canada.

Lillard, A., y Else-Quest, N. (2006). Evaluating montessori education. Science, 313, 1893-1894. doi: 10.1126/science.1132362.

Manjunath, N. K., y Telles, S. (2001). Improved performance in the Tower of London test following yoga. Indian Journal of Physiological Pharmacology, 45, 351-354.

Melby-Lervag, M., y Hulme, C. (2013). Is working memory training effective? A meta-analytic review. Developmental Psychology. 49, 270-291. doi: 10.1037/a0028228.

Raver, C. C., Jones, S. M., Li-Grining, C., Zhai, F., Bubb, K., y Pressler, E. (2011). CSRP's impact on low-income preschoolers' preacademic skills: self-regulation as a mediating mechanism. Child Development. 82, 362-378. doi: 10.1111/j.1467-8624.2010.01561.x.

Riggs, N. R., Greenberg, M. T., Kusché, C. A., y Pentz, M. A. (2006). The mediational role of neurocognition in the behavioral outcomes of a social-emotional prevention program in elementary school students: Effects of the PATHS curriculum. Prevention Science, 7, 91-102.

Rueda, M. R., Rothbart, M. K., McCandliss, B. D., Saccomanno, L., y Posner, M. I. (2005). Training, maturation, and genetic influ- ences on the development of executive attention. Proceedings of the National Academy of Sciences, USA, 102, 14931-14935.

Shipstead, Z., Redick, T. S., y Engle, R. W. (2012). Is working memory training effective? Psychological Bulletin. 138, 628-654. doi: 10.1037/a0027473.

Sonuga-Barke, E. J. S., y Halperin, J. M. (2011). Developmental phenotypes and causal pathways in attention deficit/hyperactivity disorder: potential targets for early intervention? Journal of Child Psychology and Psychiatry, 51, 368-389. doi: 10.1111/j.1469- 7610.2009.02195.x. 
Traverso, L., Viterbori, P., y Usai, M. C. (2015). Improving executive function in childhood: evaluation of a training intervention for 5-year-old children. Frontiers in psychology, 6, 525-539. doi: 10.3389/fpsyg.2015.00525.

Thorell, L. B., Lindqvist, S., Bergman Nutley, S., Bohlin, G., y Klingberg, T. (2009). Training and transfer effects of executive functions in preschool children. Developmental Science, 12, 106-113. doi: 10.1111/j.1467-7687.2008.00745.x.

Tomporowski, P. D., McCullick, B., Pendleton, D. M., y Pesce, C. (2015). Exercise and children's cognition: The role of exercise characteristics and a place for metacognition. Journal of Sport and Health Science, 4(1), 47e55. http://dx.doi.org/ 10.1016/j.jshs.2014.09.003.

van Lier, P. A., y Deater-Deckard, K. (2016). Children's Elementary School Social Experience and Executive Functions Development: Introduction to a Special Section. Journal of abnormal child psychology, 44(1), 1-6.

Volckaert, A. M. S., y Noël, M. P. (2015). Training executive function in preschoolers reduce externalizing behaviors. Trends in Neuroscience and Education, 4(1), 37-47. 
